\title{
Physiological Parameters of the Gastric Mucosal Barrier
}

\author{
HORACE W. DAVENPORT, DSc
}

An important function of the gastric mucosa is to contain within the lumen of the stomach the acid which the mucosa itself secretes. This function is expressed as the very low permeability of the mucosa to hydrogen ions. The interstitial fluid of the gastric mucosa contains sodium ions at a concentration very nearly equal to the sodium concentration of plasma, and the same property of impermeability which prevents hydrogen ions from diffusing into the lumen prevents sodium ions from diffusing from the mucosa into the lumen (1).

Capillaries of the gastric mucosa, like most other capillaries, are highly permeable to electrolytes and to small molecules of the plasma. Their permeability to plasma proteins has not been measured, and this becomes an important question when one learns how much plasma protein is shed into the gastric lumen when the mucosa is damaged. The classical means of estimating the protein concentration of interstitial fluid of an organ is to measure the protein concentration of the lymph collected from that organ. Until recently lymph has not been collected from the stomach for the reason that the arborization of lymphatic vessels draining the stomach makes cannulation difficult or impossible. Dr. Teresa Bruggeman working in my laboratory has, with great pertinacity, applied the methods of renal micropuncture to collection of gastric lymph (2). She has found that the albumin concentration of gastric lymph is about $80 \%$ of that of simultaneously collected plasma samples. Gastric lymph also contains the other plasma proteins, including fibrinogen. If in fact gastric lymph concentration of albumin does equal interstitial concentration, the high concentra-

From the Department of Physiology, University of Michigan, Ann Arbor, Michigan.

Address for reprint requests: Dr. H.W. Davenport, Department of Physiology, University of Michigan Medical Center, Ann Arbor, Michigan 48104. tion of albumin has interesting and important hemodynamic consequences, and it explains why plasma proteins are so readily shed by the injured gastric mucosa.

The property of impermeability to hydrogen and sodium ions has been conveniently called the gastric mucosal barrier (3). The events following breaking of the barrier have been repeatedly reviewed and summarized, and I will not discuss them in detail here. I will, however, describe briefly the events occurring when the barrier is broken with acid in the lumen and when it is broken without acid in the lumen.

On account of its great clinical importance, a very large amount of work has been done describing the effects of aspirin, or acetylsalicylic acid, as a barrier breaker $(4,5)$. Aspirin in its un-ionized form is relatively fat soluble, and consequently when it is dissolved in acid contents of the stomach it can diffuse through the lipids which form a large part of the membrane of the mucosal cells. Because the $\mathrm{pK}_{\mathrm{a}}$ of aspirin is 3.5 it is un-ionized in acid gastric contents, but immediately upon entering the neutral interior of the gastric mucosal cells it ionizes. This establishes a steep diffusion gradient, promoting the diffusion of the un-ionized aspirin from the lumen into the cells, and aspirin is rapidly absorbed. When aspirin is in neutral solution it is ionized and relatively insoluble in fat. Therefore, when the gastric contents are neutral, aspirin is poorly absorbed into the gastric mucosa. This fact is used in the formulation of aspirin-containing products; if the product is adequately buffered to near neutrality, its acetylsalicylate will not be absorbed in any appreciable quantity into the gastric mucosa.

For reasons I do not understand, aspirin, once it enters the mucosal cells, damages them and breaks the gastric mucosal barrier (4). 
The effects of acid back-diffusion through a broken barrier can be illustrated by experiments in which the gastric mucosa is irrigated with a solution of aspirin in $100 \mathrm{mN} \mathrm{HCl}$. During a control period in which the mucosa is irrigated with $100 \mathrm{mN} \mathrm{HCl}$ alone virtually nothing happens. Because the barrier is intact, few hydrogen ions diffuse into the mucosa, and few sodium ions diffuse from the mucosa into the lumen. The mucosa sheds no protein-containing fluid. When the acid solution contains aspirin the barrier is broken. The hydrogen ions diffuse into the mucosa, and a large amount of sodium leaves the mucosa. Acid back-diffusion into the mucosa has many consequences, many still poorly understood (6). Acid stimulates the intramural plexuses of the stomach and by this means stimulates the secretion of pepsinogen. Motility is also stimulated, and the resulting strong contractions are apparently associated with the sensation of pain. Histamine is liberated from mucosal stores, and histamine-forming capacity is increased. As a result, histamine reaches a high concentration in mucosal interstitial fluid and exerts its characteristic actions upon the mucosa. Secretion of acid is stimulated, and we have the apparently paradoxical situation that at a time when acid is diffusing into the mucosa the amount of acid in luminal contents may actually be increasing as the result of copious secretion. Histamine affects the mucosal blood vessels, promoting vasodilatation and increased capillary filtration. As a result, fluid rapidly enters the interstitial spaces. In normal circumstances, fluid entering interstitial spaces by filtration is at once secreted into the lumen, but during injury fluid accumulates. Some of this edema fluid is filtered into the lumen, carrying with it sodium, chloride, and bicarbonate. Of course, we never find the bicarbonate, for it reacts with acid and disappears as carbon dioxide. Histamine may increase the permeability of mucosal capillaries to plasma proteins and make the walls of the vessels more sticky. As the result of filtration of plasma proteins from the capillaries, the interstitial fluid which is filtered into the lumen contains plasma proteins. Finally, acid may rupture mucosal capillaries, and interstitial hemorrhage and frank bleeding follow. Shed hemoglobin is so obvious that bleeding is easily detected, but unless one looks for them one does not see the plasma proteins beneath the hemoglobin. In my experience, when I have looked for plasma proteins I have found the hematocrit of the shed blood to be no greater than $10 \%$, meaning that much more plasma than red cells is being lost. I have also found that cholinergic stimulation exacerbates bleeding, perhaps because acetylcholine promotes contraction of gastric musculature, and this in turn causes venous engorgement.

In contrast, the results are quite different when the mucosa is irrigated with the same concentration of aspirin in a solution of $\mathrm{pH} 3$, or $1 \mathrm{mN} \mathrm{HCl}$. In this solution aspirin is largely un-ionized, and it enters the mucosa as rapidly as from $100 \mathrm{mN} \mathrm{HCl}$. It also breaks the gastric mucosal barrier. However, because the concentration of hydrogen ions is low in a solution at $\mathrm{pH} 3$, there is little acid to diffuse back into the mucosa, and none of the consequences of acid back-diffusion occurs. There is no stimulation of secretion, no shedding of interstitial fluid, no loss of plasma proteins, and no bleeding. This result clearly illustrates the importance of acid back-diffusion as distinct from barrier breaking.

Blood flow during gastric mucosal injury may go up, or it may go down. Histamine in interstitial fluid causes vasodilatation. It is likely that reflexes mediated by the intramural plexuses also cause vasodilatation and increase blood flow. In addition, active hyperemia accompanies secretion. On the other hand, blood flow may fall almost to zero, particularly in the more superficial vessels (8). The reason is that histamine induces the triple response, and capillary stasis occurs. The small blood vessels are completely plugged with red blood cells.

There has been a lively debate over the role of ischemia in causing gastric mucosal damage. It seems reasonable to suppose that a profound decrease in blood flow to the gastric mucosa must damage the mucosa, but this is not true in the dog (9) or the monkey (10). We do not know what is the situation in man. The experimental results show that the gastric vasculature escapes from strong vasoconstrictive influences, and the moral of this is that vasoconstriction may not be present when you think it ought to be. Furthermore, even when vasoconstriction is induced by massive infusions of vasopressin or norepinephrine, stimulation of the mucosa with histamine or with bethanechol and pentagastrin induces vasodilatation. Active hyperemia, as we try to teach medical students, overcomes vasoconstriction.

Although much work has given us some understanding of damage and its consequences, we know little about the equally important topic of mucosal repair. This is a very rapid process, for a severely damaged gastric mucosa can recover its barrier 
function in a couple of hours. It can do this even if it is bathed by acid and is bleeding. When the mucosa is damaged, most of the surface epithelial cells desquamate, but repair of barrier function can occur long before the lost cells have been replaced. We need a systematic study of the factors influencing, for good or ill, the ability of the mucosa to regain its barrier function. What drugs and what hormones promote or impair that ability?

\section{REFERENCES}

1. Davenport HW, Warner HA, Code CF: Functional significance of gastric mucosal barrier to sodium. Gastroenterology $47: 142-152,1964$

2. Bruggeman T: Plasma proteins in gastric lymph. Gastroenterology 68:1204-1210, 1975

3. Code CF, Scholer JF, Orvis AL: Barrier offered by gastric mucosa to absorption of sodium. Am J Physiol 183:604, 1955
4. Davenport HW: Gastric mucosal injury by fatty and acetylsalicylic acids. Gastroenterology 46:245-253, 1964

5. Davenport HW: Gastric mucosal hemorrhage in dogs: Effects of acid, aspirin, and alcohol. Gastroenterology 56:439449, 1969

6. Davenport HW: Back diffusion of acid through the gastric mucosa and its physiological consequences. Progress in Gastroenterology, Vol 2. Glass GJ (ed). New York, Grune and Stratton, 1970

7. Davenport HW: Fluid produced by the gastric mucosa during damage by acetic and salicylic acids. Gastroenterology 50:487-499, 1966

8. Davenport HW, Munro D: Aminopyrine clearance in the damaged gastric mucosa: Reconciliation of conflicting data. Gastroenterology 65:512-514, 1973

9. Davenport HW, Barr LL: Failure of ischemia to break the dog's gastric mucosal barrier. Gastroenterology 65:619-624, 1973

10. Ritchie WP Jr: Effect of hemorrhage on electrical and $\mathrm{pH}$ gradients in the intact stomach of the subhuman primate. Gastroenterology 67:259-263, 1974 